\title{
Algèbres de Banach ultramétriques, algèbres de Krasner et algèbres de Krasner-Tate
}

\author{
Mémoires de la S. M. F., tome 39-40 (1974), p. 39-54 \\ <http://www.numdam.org/item?id=MSMF_1974_39-40_39_0>
}

CC Mémoires de la S. M. F., 1974, tous droits réservés.

L'accès aux archives de la revue « Mémoires de la S. M. F. » (http://smf. emath.fr/Publications/Memoires/Presentation.html) implique l'accord avec les conditions générales d'utilisation (http://www.numdam.org/conditions). Toute utilisation commerciale ou impression systématique est constitutive d'une infraction pénale. Toute copie ou impression de ce fichier doit contenir la présente mention de copyright.

\section{Numdam}


Bull. Soc. math. France,

Mémoire 39-40, 1974, p.39-54

\section{ALGEBRES DE BANACH ULTRAMETRIQUES}

ALGEBRES DE KRASNER

ET

ALGEBRES DE KRASNER-TATE

Alain ESCASSUT

\section{INTRODUCTION}

Nous commencerons par étudier les propriétés algébriques d'une algèbre H(D) d'éléments analytiques au sens de Krasner [14] sur une partie D d'un corps $K$ valué non archimédien complet, algébriquement clos, en caractérisant ces propriétés par la forme géométrique de D. Nous verrons notamment que $H(D)$ est principale si et seulement si $D$ est un ouvert qui ne possède pas certains types de filtre que nous nommerons T-filtres et qui jouent également un rôle important dans l'étude des domaines analytiques au sens de Motzkin $[6,7,18,20]$.

Certaines algèbres $H(D)$ admettent des idéaux maximaux de codimension infinie et nous étudierons enfin le corps quotient de cette algèbre par un tel idéal.

Nous étudierons ensuite le rapport existant entre la théorie des algèbres de Tate [13] et [22] et la théorie des algèbres de Krasner [11] et [14]. Nous nommerons algèbre de Krasner-Tate toute algèbre isomorphe à la fois à une algèbre de Krasner et à une algèbre de Tate et nous caractériserons ses propriétés en tant qu'algèbre de Krasner d'une part, et en tant qu'algèbre de Tate d'autre part. Nous verrons, entre autre, que les algèbres de Krasner-Tate intègres sont des algèbres d'éléments analytiques au sens de Krasner sur un fermé borné $D$ de $K$ dont les trous sont en nombre fini. Ce sont aussi les algèbres de Tate de la forme :

$$
\frac{K\{T, X\}}{(P(X)-T Q(X)) K\{T, X\}}
$$

où $P$ et $Q$ sont deux polynômes en $X$ possédant certaines propriétés. Nous caractériserons enfin les algèbres de Krasner-Tate parmi les algèbres de Banach à l'aide de leurs propriétés algébriques et topologiques.

A cette occasion nous étudierons certaines propriétés spectrales des algèbres de Banach ultramétriques et nous construirons différents exemples permettant de comparer ces propriétés avec celles des $\mathbb{C}$-algèbres de Banach et de voir quelles relations existent entre la norme d'une $K$-algèbre de Banach ultramétrique et sa 
semi-norme spectrale.

Nous conclurons cette étude en caractérisant les algèbres de Tate de degré 1 parmi les algèbres de Banach.

On trouvera les démonstrations des résultats du $\S .1$ dans [5], celles des résultats des $\$ .2,3,4$ dans [23].

1. Les Algèbres de Krasner.

Soit $K$ un corps valué non archimédien, complet, algébriquement clos et soit $x \longrightarrow|x|$ sa valeur absolue. Soit $D$ un fermé borné de $K, K(D)$ la K-algèbre unitaire des fractions rationnelles sans pôle dans $D . K(D)$ est normée par la norme de la convergence uniforme, mais non complète. Soit $H(D)$ la K-algèbre de Banach complétée de $K(D)$. Les éléments de $H(D)$ sont appelés éléments analytiques sur $D$ au sens de Krasner $[14]$.

Nous nous posons la question suivante : à quelles conditions portant sur D l'algèbre $H(D)$ sera-t-elle noethérienne ou intègre? L. Gruson a montré [11] (avec quelques restrictions) que si $\mathrm{H}(\mathrm{D})$ est noethérienne, alors elle est principale.

Nous essaierons de généraliser ces résultats et surtout de caractériser les fermés bornés $D$ de $K$ tels que $H(D)$ soit noethérienne ou tels que $H(D)$ soit intègre.

Ceci nécessite l'introduction de notions nouvelles.

Définition 1. Un fermé borné $D$ est dit infraconnexe si quels que soient a et $b \in D$, l'image par l'application $x \rightarrow|x-a|$ de l'ensemble des $x \in D$ tels que $|x-a| \leqslant|a-b|$ est dense dans l'intervalle $[0,|a-b|]$ de $R$.

Exemple. Tout quasi-connexe [14] est infraconnexe. Il résulte de la définition qu'une réunion enchaînée d'infraconnexes est infraconnexe et que la relation $R$ définie sur D par : $x \Omega y$, si et seulement s'il existe une partie infraconnexe de $D$ contenant $\mathrm{x}$ et $\mathrm{y}$ est une relation d'équivalence.

Définition 2. Les classes d'équivalence de la relation $R$ sont appelées composantes infraconnexes de $D$.

D'autre part, I'infraconnexité de $D$ confère à $H(D)$ la propriété suivante :

Proposition 1. Les seuls idempotents de l'algèbre de Banach $H(D)$ sont 0 et 1 si et seulement si D est infraconnexe.

Pour simplifier le problème posé, nous étudierons d'abord H(D) lorsque $D$ est infraconnexe, puis lorsque ce problème sera résolu, nous étudierons le cas général. 
Le fait que $H(D)$ ne soit pas noethérienne lorsque $D$ est infraconnexe fait apparaître sur $D$ I'existence de certains filtres que nous appellerons T-filtres. Auparavant, nous devons définir, certaines notions destinées à les introduire.

Définition 3. Soit a $\epsilon K$, soit $r$ un nombre positif. On appelle d.n.c. (disque non circonférencié) de centre a, de rayon $r$, l'ensemble des $x \in \mathrm{K}$ tels que $|x-a|<r$.

On appelle cercle de centre a de rayon $r \underline{\text { l'ensemble des }} \times \in \mathrm{K}$ tels que $|x-a|=r$.

On appelle classe d'un cercle $C$ tout d.n.c. dont le rayon est égal au rayon du cercle, et inclus dans $C$.

Définition 4. Soit $D$ un infraconnexe de $K$, de diamètre $R$. Soit $A$ un d.n.c. de rayon $r \leqslant R$, tel que $A \cap D \neq A$. Soit $q$ un entier $\geqslant 0$. Soit $\left.\varepsilon_{(}^{\prime} A, q\right)$ I'ensemble des poly nômes de degré $q$ dont tous les zéros appartiennent à $A-(A \cap D)$. Soit $P \in E(A, q)$, posons alors

$$
\begin{array}{ll}
\left\|\frac{1}{P}\right\|_{A \cap D}=\sup _{x \in A \cap D}\left|\frac{I}{P(x)}\right| & \text { si } A \cap D \neq \varnothing . \\
\left\|\frac{1}{P}\right\|_{A \cap D}=\frac{1}{r^{q}}, & \text { si } A \cap D=\varnothing .
\end{array}
$$

Posons enfin

$$
Y(A, q)=r^{q} \inf _{P \in \varepsilon(A, q)}\left\|\frac{1}{P}\right\|_{A \cap D}
$$

Définition 5. Soit $a_{m}$ une suite de points de $D$ telle que la suite $a_{m}=\left|a_{m+1}-a_{m}\right|$ soit strictement décroissante, de limite non nulle a. Soit $\mathrm{C}_{\mathrm{m}}$ le cercle de centre a, de rayon $\mathrm{d}_{\mathrm{m}}$ '.

Soit $\Delta_{\mathrm{m}} I^{\prime}$ ensemble des $\mathrm{x} \in \mathrm{D}$ tels que $\left|\mathrm{x}-\mathrm{a}_{\mathrm{m}}\right| \leqslant \mathrm{d}_{\mathrm{m}}$.
Soit $\Delta=\bigcap_{\mathrm{m}=1}^{\infty} \Delta_{\mathrm{m}}$. Soit $\mathrm{D}_{\mathrm{m}}=\Delta_{\mathrm{m}}-\Delta$.

On appelle filtre associé à la suite $a_{m}$ le filtre de $D$ admettant pour base la suite $D_{m} \cdot$ On appelle suite de cercles $C_{m}$ associée à la suite a ${ }_{m}$ la suite des cercles $C_{m}$ définis ci-dessus.

Définition 6. On appelle T-filtre décroissant d'un infraconnexe $D$, un filtre associé à une suite $a_{m}$ telle que la suite des cercles $C_{m}$ associée possède la propriété suivante :

Quel que soit $m \geqslant \Gamma, c_{m}$ admet un nombre fini $k(m)$ de classe $\Gamma_{m, i}$ $[i=1, \ldots, k(m)]$ vérifiant $\left(\Gamma_{m, i}\right) \cap D \neq \Gamma_{m, i}$, chacune étant ssociée à un entier $q_{m, i}$ tel que les suites $r_{m}, d_{m}, q_{m}$, définies par 


$$
r_{m}=\sup _{i=1, \ldots, k(m)} r\left(r_{m, i}, q_{m, i}\right) ; q_{m}=\sum_{i=1}^{k(m)} q_{m, i} \text { et } a_{m}=\left|a_{m+1}-a_{m}\right|
$$

vérifient la relation

$$
\lim _{n \rightarrow \infty}\left(r_{m}\left[\prod_{j=1}^{m-1}\left(\frac{d_{m}}{d_{j}}\right)^{q}\right]\right)=0
$$

Définition 7. Un filtre $F$ de $D$ est appelé T-filtre crojssant s'il existe un infraconnexe $D^{\prime}$, un T-filtre décroissant $F^{\prime}$ de $D$ et une inversion $J$ de pôle $a \in \int_{K} D \cap D^{\prime}$ telle que $J(D)=D^{\prime}$ et $J(\mathcal{F})=F^{\prime}$.

Définition 8. On appelle plage d'un T-filtre décroissant $F$ de $D$ associé à une suite a et on note $P(\mathcal{F})$ l'ensemble $\Delta \cap D,(\Delta$ étant défini comme à la définition 5).

De même on appelle plage d'un T-filtre croissant fi (inverse par J d'un T-filtre décroissant $F$ ) et on note $P(\mathcal{F})$ I'ensemble $J\left(\rho\left(f^{\prime}\right)\right)$; il est évident que $\rho(F)$ ne dépend pas de 1 'inversion I considérée.

D'autre part, nous noterons $C(F) I$ 'ensemble $D-P(F)$.

Définition 9. Deux T-filtres de $D, F_{1}$ et $F_{2}$ sont dits complémentaires si $P\left(\mathcal{F}_{1}\right) \cup P\left(\mathcal{F}_{2}\right)=D$.

Nous sommes maintenant en mesure d'énoncer les conclusions.

Théorème 1. Soit $D$ une partie de $K$. Alors $H(D)$ est une algèbre de Banach unitaire intègre si et seulement $s i$ est infraconnexe sans couple de T-filtres complémentaires.

Théorème 2. Soit $H(D)$ une algèbre de Banach unitaire sans indempotents autres que 0 et 1. Alors les conditions suivantes sont équivalentes :

a) Tout idéal est fermé dans $H(D)$;

b) $\mathrm{H}(\mathrm{D})$ est noethérienne ;

c) $H(D)$ est principale ;

d) Tout idéal est engendré par un polynôme dont les zéros sont intérieurs à $D$;

e) D est ouvert et sans T-filtre. 
Théorème 3. Soit $D$ un fermé borné de $K$. Alors les assertions suivantes sont équivalentes :

a) $H(D)$ est une algèbre de Banach unitaire noethérienne ;

b) Tout idéal de $H(D)$ est principal ;

c) Tout idéal de H(D) est fermé ;

d) Les composantes infraconnexes de $D$ sont en nombre fini, et chacune est ouverte et sans T-filtre ou réduite à un point.

De plus, si ces conditions sont réalisées, soient $A_{1}, \ldots, A_{n}$ les composantes infraconnexes de D. Alors $H(D)$ est isomorphe à

$$
H\left(A_{1}\right) \times H\left(A_{2}\right) \times \ldots \times H\left(A_{n}\right) .
$$

Nous pouvons également apporter des précisions intéressantes au sujet de la nature des idéaux maximaux de $H(D)$, mais devons pour cela introduire deux notions nouvelles.

Définition 10. Nous dirons qu'un T-filtre décroissant Fest imbriqué dans un T-filtre croissant $F^{\prime}$, s'il existe un élément $A$ de $F_{\text {inclus dans }} e\left(F^{\prime}\right)$.

Définition 11. Nous dirons qu'une famille de T-filtres $\tau=\left(F_{i}\right)_{i} \in$ I est une T-famille si la famille $C\left(\hat{H}_{i}\right)_{i} \in$ I est une partition de $D$ et si tout T-filtre décroissant de $D$ qui n'est pas imbriqué dans un élément de $\tau$ est complémentaire de tout élément croissant de $\tau$.

Remarque. Il est clair qu'une T-famille contient au plus un élément décroissant. Elle contient un seul élément sị et seulement si sa plage est vide.

Nous pouvons maintenant conclure.

Proposition 2. Soit une T-famille $\tau$ de $D$ et soient $F_{1}$ et $F_{2} \in \tau$. Alors $I\left(F_{1}\right)=I\left(F_{2}\right)$. De plus, $I\left(f_{1}\right)$ est maximal.

Ce résultat permet d'associer à toute T-famille $\tau$ un idéal maximal que nous noterons $I(\tau)$. Puis on démontre que cette application est une bijection de I'ensemble des $\mathrm{T}$-familles de $\mathrm{D}$ sur le spectre maximal de codimension infinie de $\mathrm{H}(\mathrm{D})$ et on obtient finalement le théorème suivant :

Théorème 4. Soit $D$ une partie de $K$ telle que $H(D)$ soit une algèbre sans idempotent autre sur 0 et 1 . Alors les idéaux maximaux de $\mathrm{H}(D)$ sont :

ou bien principaux, de codimension 1 , et de la forme $I(a)$, où a est un point intérieur à D; 
ou bien de type fini, de codimension 1 , et de la forme $I(a)$, où a est un point de $D$ non intérieur à $D$;

ou bien de type et codimension infinis et de la forme $I(\tau)$ où $\tau$ est une T-famille de D.

Exemple de quotient d'une algèbre $H(D)$ par un idéal maximal $\pi$ de codimension infinie.

Soit $\Gamma=\mathrm{H}(D) / \mathcal{M}$. Le corps $\Gamma$ est une algèbre de Banach avec la norme quotient $\|\cdot\|$.

D'autre part, $\Gamma$ peut être muni de la façon suivante d'une valeur absolue naturelle qui prolonge celle de K. Soit $\varphi$ la surjection canonique de H(D) sur $\Gamma$. Soit $\tau$ une $T$-famille telle que $\mathcal{M}=I(\tau)$ et soit $\hat{F} \in \tau$. Soit a $\in \mathcal{C}(\hat{F})$ et soit $r$ le diamètre de $e(\hat{\mathcal{H}})$. Soit $\theta \in \Gamma$ et soit $f \in H(D)$ tel que $\varphi(f)=\theta$. Il est clair que $|f(x)|$ a une limite quand $|x-a|$ tend vers $r$ et que cette limite ne dépend pas de $f$ tel que $\varphi(f)=\theta$. On peut donc définir sur $\Gamma$ la fonction $|$.$| par$ $\theta \rightarrow|\theta|=\lim _{|x-a| \rightarrow r}|f(x)|[\varphi(f)=\theta]$. Il est évident que $|$.$| est une valeur absolue$ sur $\Gamma$ et que $|\theta| \leqslant\|\theta\|$ quel que soit $\theta \in \Gamma$.

Etudions $\Gamma$ lorsque $D$ a la forme suivante. Soit $|K|$ l'ensemble des $|x|, x \in K$. Soit $R \in|K|(R>0)$ et $\rho \in|K|$ tel que $\rho \leqslant R$. Soit $r_{n}$ une suite de $|K|$ croissante de limite $R \in R$ et soit $\rho_{\mathrm{n}}$ une suite de $|K|$, de limite $\rho$ telle que $0<\rho_{n} \leqslant r_{n}$ quel que soit $n$. Soit $D l^{\prime}$ ensemble des $x \in K$ tels que $|x|<R$ et $\mid x-a_{n} j \geqslant \rho_{n}$ quel que soit $n$. On sait d'après [5] que $D$ admet un T-filtre croissant à plage vide si et seulement $s i$ il existe une suite d'entiers $p_{n} \geqslant 0$ tels que

$$
\lim _{n \rightarrow \infty} p_{n}\left(\log \rho_{n}-\log r_{n}\right)+\sum_{m=1}^{n-1} p_{m}\left|\log r_{n}-\log r_{m}\right|=+\infty \text {. }
$$

De plus, on a vu que quel que soit $\rho \in[0, R]$, il existe une suite $r_{n}$ et une suite $\rho_{n}$ définies comme ci-dessus, telles que cette condition (1) soit satisfaite. Supposons-la donc réalisée et comparons de façon plus précise la norme d'algèbre de Banach quotient et la valeur absolue définies sur $\Gamma$. En utilisant le théorème de Banach et en montrant que si $\rho=0$, l'ensemble des éléments topologiquement nilpotents de $\Gamma$ est non borné, on obtient le théorème suivant :

Théorème 6. Soit $\ell=\rho / R$. Alors on a les propositions suivantes :

a) $\|\theta\|=|\theta|$ quel que soit $\theta \in \Gamma$ si et seulement si $l=1$;

b) la valeur absolue et la norme quotient définissent la même topologie sur $r$ 
si et seulement si $0<\ell \leqslant 1$;

c) la topologie de la norme quotient est strictement plus fine que celle de la valeur absolue et $\Gamma$ n'est pas complet pour cette valeur absolue si et seulement si $l=0$.

\section{Algèbres de Krasner-Tate.}

Soit un corps $K$ valué non archimédien complet. Soit $A=\frac{K\left\{x_{1}, \ldots, x_{n}\right\}}{I}$ une algèbre topologiquement de type fini où $\mathrm{K}\left\{\mathrm{x}_{1}, \ldots, \mathrm{x}_{n}\right\}$ est une extension topologiquement pure de $K$ et $I$ un idéal de $K\left\{x_{1}, \ldots, X_{n}\right\}$. Nous savons grâce à la proposition (4.4) de [22], qu'une algèbre de Banach A est topologiquement de type fini sur $\mathrm{K}$ si et seulement si c'est une extension entière finie d'une extension topologiquement pure de K. D'autre part, nous savons, grâce à la proposition (4.5) de [22] que A est noethérienne et que tout idéal maximal est de codimension finie. On peut à ce sujet établir un théorème des zéros de Hilbert pour une extension topologiquement pure dont on déduit que si $\mathrm{K}$ est algébriquement clos, le spectre maximal de $\mathrm{A}$ est en bijection de façon naturelle avec une partie de $U^{n}$, U étant l'ensemble des $x \in K$ tels que $|x| \leqslant 1,(n \in \mathbb{N}$ ).

Définition 1. Nous dirons qu'une algèbre de Banach A est une algèbre de KrasnerTate si elle est isomorphe à la fois à une algèbre topologiquement de type fini sur $\mathrm{K}$ et à une algèbre $H(D)$ telle que $\mathrm{D}$ soit un fermé borné infini de $\mathrm{K}$.

Nous noterons $|K| l$ 'ensemble des $|\xi|, \xi \in \in K$.

Définition 2. Nous dirons qu'un infraconnexe fermé borné est calibré si son diamètre appartient à $|K|$ et si le diamètre de chacun de ses trous appartient à $|K|$. Nous dirons plus généralement qu'un fermé borné est calibré si c'est une réunion finie d'infraconnexes calibrés.

Définition 3. Nous dirons qu'une partie infinie $D$ de $K$ est ultracirconférenciée si c'est une réunion finie de fermés bornés calibrés disjoints dont les trous sont en nombre fini.

On peut maintenant conclure :

Théorème 1. Soit D un fermé borné. Alors les propositions suivantes sont équivalentes :

a) D est ultracirconférencié ;

b) il existe une fraction $t=\frac{P}{Q} \in K(X)$ sans pôle dans $D$, telle que $\operatorname{deg}(P)>\operatorname{deg}(Q)$, 
$t(D)=U$ et telle que l'algèbre $K\{t\}$ des séries formelles restreintes en $t$ munie de la norme $\|\cdot\|_{D}$ de $H(D)$ soit isométriquement isomorphe à une extension topologiquement pure de degré 1 et satisfasse $H(D)=K\{t\}[x]$ où $x$ est l'application identique de $\mathrm{D}$;

c) $H(D)$ est une algèbre de Krasner-Tate.

De plus, dans le cas où $H(D)$ est non dégradée, $H(D)$ est une algèbre de Krasner-Tate si et seulement s'il existe une fraction $t$ satisfaisant l'assertion $b$ ) et vérifiant également $D=t^{-1}(U) \quad\left(t^{-1}(U)\right.$ étant l'ensemble des $\zeta \in K$ tels que

$|t(\zeta)| \leqslant 1) .(H(D)$ est dite non dégradée si $D$ est ouvert).

Théorème 2. Soit $D$ un ouvert ultracirconférencié inclus dans $U$ et soit $t$ une fraction possèdant les propriétés énoncées dans l'assertion b) du théorème 1. Alors le spectre de $H(D)$ considéré comme algèbre topologiquement de type fini sur $K$ est l'ensemble des couples $(t(\mu), \mu) \mu \in D$.

Nous utiliserons fréquemment les définitions suivantes :

Définition 4. Soit $A$ un anneau et soient $a, b \in A$. On dit que a et $b$ sont fortement étrangers dans $A$ si $a A+b A=A$.

Définition 5 . Soient $D$ et $D^{\prime}$ deux fermés bornés de $K$ tels que $H(D)$ et $H^{\prime}\left(D^{\prime}\right)$ soient deux algèbres de Banach isomorphes. Nous dirons que $D$ et $D^{\prime}$ sont isomorphes.

Théorème 3. Soit $H(D)$ une algèbre de Krasner-Tate non dégradée et soit $D^{\prime}$ isomorphe à $D$ tel que $D^{\prime} C U$. Soient $P$ et $Q$ deux polynômes fortement étrangers de $K[X]$ satisfaisant

a) $P$ est unitaire ;

b) $\operatorname{deg}(P)>\operatorname{deg}(Q)$;

c) $1=\|P\| \geqslant\|Q\|$ pour la norme canonique de $K[X]$.

d) La fraction $t=\frac{P}{Q} \in K\left(D^{\prime}\right)$ satisfait $t\left(D^{\prime}\right)=U, D^{\prime}=t^{-1}(U)$.

Alors $H(D)$ est isomorphe à l'algèbre topologiquement de type fini $K\{T, X\}$ où $K\{T, X\}$ est une extension topologiquement pure de degré 2 . $(P(X)-T Q(X)) K\{T, X\}$
Réciproquement, soient $P$ et $Q$ deux polynômes fortement étrangers de $K[X]$ satisfaisant a), b), c). Soit $t=\frac{P}{Q} \in K(X)$ et soit $D=t^{-1}(U)$. Alors l'algèbre topologiquement de type fini

$$
\frac{K\{T, X\}}{P(X)-T Q(X)) K\{T, X\}}
$$


est isomorphe à $H(D)$.

Théorème 4. Soit $H(D)$ une algèbre de Krasner-Tate. Soit $D^{\prime}$ isomorphe à $D$ tel que $D^{\prime} \subset U$, soient $D_{1}, \ldots, D_{n}$ les composantes infraconnexes de $D^{\prime}$ qui ne sont pas réduites à un point et soient $a_{1}, \ldots, a_{n}$ les points isolés de $D^{\prime}$. Pour toute famille de couples $\left(P_{i}, Q_{i}\right) \quad(1 \leqslant i \leqslant n)$ de polynômes fortement étrangers de $K[X]$, on notera a), b), c), d), e), f) les propriétés suivantes :
a) la fraction $t_{i}=\frac{P_{i}}{Q_{i}}$ satisfait $t_{i}\left(D_{i}\right)=U, D_{i}=t_{i}^{-1}(U),(1 \leqslant i \leqslant n)$;
b) $P_{i}$ est unitaire $(1 \leqslant i \leqslant n)$;
c) $\operatorname{deg}\left(P_{i}\right)>\operatorname{deg}\left(Q_{i}\right)(1 \leqslant i \leqslant n)$;
d) $1=\left\|P_{i}\right\| \geqslant\left\|Q_{i}\right\|$ pour la norme canonique de $K\{x\},(1 \leqslant i \leqslant n)$;
e) $P_{i}-T Q_{i}$ est irréductible dans $K\{T, X\},(1 \leqslant i \leqslant n)$;
f) $P_{i}-T Q_{i}$ et $P_{j}-T Q_{j}$ sont fortement étrangers dans $K\{T, X\}$, quels que soient $i \neq j$.

Soit une famille de couples de polynômes fortement étrangers de $K[X]$, $\left(P_{i}, Q_{i}\right)(1 \leqslant i \leqslant n)$ satisfaisant $\left.\left.\left.a\right), b\right), c\right)$. Soient $b_{1}, \ldots, b_{m} \in U$.

Alors la famille $\left(P_{i}, Q_{i}\right)(1 \leqslant i \leqslant n)$ satisfait $\left.\left.\left.d\right), e\right), f\right) ; H\left(D_{i}\right)$ est isomorphe à $\frac{K\{T, X\}}{\left(P_{i}(X)-T Q_{i}(X)\right) K\{T, X\}} ; H(D)$ est isomorphe à

$$
\frac{K\{T, X\}}{\prod_{j=1}^{m}\left[\left(x-a_{j}\right) K\{T, X\}+\left(T-b_{j}\right) K\{T, x\}\right] \prod_{j=1}^{i n}\left(P_{i}(x)-T Q_{i}(x)\right) K\{T, X\}}
$$

Réciproquement, soit $A$ une algèbre topologiquement de type fini de la forme ci-dessus où $\left(a_{j}, b_{j}\right) \in U \times U(1 \leqslant j \leqslant m)$ et où $\left(P_{i}, Q_{i}\right)$ est un couple de polynômes fortement étrangers de $K[X] \quad(1 \leqslant i \leqslant n)$ satisfaisant $b), c), d), e), f)$. Soit $t_{i}=\frac{P_{i}}{Q_{i}} \in K(X) ;$ soit $D_{i}=t_{i}^{-1}(U) ;$ soit $D=\left\{a_{1}, \ldots, a_{m}\right\} U\left(\bigcup_{i=1}^{n} D_{i}\right)$.

Alors A est i somorphe à $H(D)$. 


\section{Spectre algébrique et idempotents associés.}

Définitions et notations. Soit $\mathrm{E}$ un corps algébriquement clos et $\mathrm{A}$ une $\mathrm{E}$-algèbre Nous appellerons spectre algébrique d'un élément $x \in A$ I'ensemble des $\lambda \in E$ tels que $x-\lambda$ appartienne à un idéal maximal de codimension 1 de $A$. Nous dirons que $x$ est de classe algébrique si son spectre est égal à son spectre algébrique. On notera $\operatorname{sp}_{A}(x)$ le spectre de $x$ et $\operatorname{sa}_{A}(x)$ son spectre algébrique.

Remarque. On a évidemment $\mathrm{sa}_{\mathrm{A}}(\mathrm{x}) \subseteq \mathrm{sp}_{\mathrm{A}}(\mathrm{x})$ pour tout $\mathrm{x} \in \mathrm{A}$.

Rappelons qu'une K-algèbre de Banach A dont l'un au moins des idéaux maximaux est de codimension 1 admet une semi-norme spectrale $\|\cdot\|$ sp définie par $\|f\|_{s p}=\sup _{\sigma \in \hat{E}}(\sigma(f))$ où $\mathfrak{S}$ désigne l'ensemble des homomorphismes de A sur $\mathrm{K}$.

Nous dirons qu'une K-algèbre de Banach ultramétrique $A$ dont la norme est notée $\|\cdot\|$ possède la propriété "P" si:

(P) elle admet une semi-norme spectrale $\|\cdot\|_{\text {sp }}$ telle que $\|f\|_{\text {sp }}=\lim _{n \rightarrow \infty} \sqrt[n]{\| f^{n}} \|$ pour tout $f \in A$.

Remarque. L'algèbre A possède la propriété "P" si et seulement si elle admet une semi-norme spectrale telle que $\|f\|_{s p}<1$ implique $\lim _{n \rightarrow \infty}\left\|f^{n}\right\|=0$.

La notion de frontière analytique définie dans [1] pour un quasi-connexe se généralise immédiatement pour un infraconnexe ainsi que la proposition 1 de [1] . Alors on a le théorème suivant :

Théorème 1. Soit $A$ une K-algèbre de Banach ultramétrique unitaire possédant la propriété "P". Alors l'enveloppe de $\mathrm{sp}_{A}(f)$ est égale à celle de $s a_{A}(f)$ pour tout $f \in A$.

De plus, si les composantes infraconnexes de $\mathrm{sp}_{\mathrm{A}}(f)$ sont en nombre fini et si on les note $D_{1}, \ldots, D_{n}$, alors $D_{i} \cap s a_{A}(f)$ est une frontière analytique de $D_{i}$. Définition. Soit $E$ un corps algébriquement clos et soit A une E-algèbre. Soit $x \in A$ et soit $\Delta$ une partie de $\operatorname{sp}_{A}(x)$. On appelle idempotent associé à $x$ et à $\Delta$ tout idempotent de classe algébrique u $\in$ A tel que $\varphi(u)=1$ pour tout homomorphisme $\varphi$ de $A$ sur $K$ tel que $\varphi(x) \in \Delta$ et tel que $\varphi(u)=0$ pour tout homomorphisme $\varphi$ de $A$ sur $K$ tel que $\varphi(x) \notin \Delta$. Grâce au théorème 1 du $\$ 2$ on établit le théorème 2. 
Théorème 2. Soit $A$ une K-algèbre de Banach ultramétrique unitaire qui possède 18 propriété "P". Si les composantes infraconnexes du spectre d'un élément $x \in$ A sont en nombre fini $n$, et si l'on note $D_{1}, \ldots, D_{n}$ ces composantes infraconnexes; alors il existe une famille d'idempotents $u_{i}$ associés à $x$ et à $D_{i}(1 \leqslant i \leqslant n)$ telle que $u_{i} u_{j}=0$ pour tout $i \neq j$ et $\sum_{i=1}^{n} u_{i}=1$.

Corollaire. Soit A une K-algèbre de Banach ultramétrique unitaire dont les idempotents sont en nombre fini $q$, et qui possède la propriété "P". Alors pour tout $x \in A$, le nombre des composantes infraconnexes de $\operatorname{sp}_{A}(x)$ est majoré par $q$.

Remarque. Le corollaire est vrai en particulier si A est une algèbre de Banach ultramétrique noethérienne qui possède la propriété "P".

Dans le cas où A contient une sous-algèbre dense de type fini, on a les deux résultats suivants.

Théorème 3. Soit A une K-algèbre de Banach ultramétrique unitaire possédant la propriété " $P$ " et contenant une sous $K$-algèbre $B$ de type fini principale et dense dans A. Alors tout élément de $B$ est de classe algébrique.

Théorème 4. Soit A une K-algèbre de Banach ultramétrique unitaire noethérienne possédant la propriété "P".

Soit $x \in A$ tel que $P(x) \neq 0$ pour tout polynôme non nul $P(X) \in K[X]$. Alors $\operatorname{sp}_{A}(x)$ est une partie infinie de $K$.

L'exemple 1 que nous allons construire montre que le théorème 4 n'est pas trivial.

Exemple 1. L'application $\|\cdot\|$ définie sur $K[X]$ par $\left\|\sum_{i=0}^{n} a_{i} x^{i}\right\|=\sup _{0 \leqslant i \leqslant n} \frac{\left|a_{i}\right|}{1+i}$ est une norme de $K$-algèbre et l'algèbre de Banach $A$ complétée de $K[X]$ pour cette norme est un anneau local qui possède la propriété "P" et tel que $\operatorname{sp}_{A}(X)=\{0\}$.

On sait que pour toute algèbre $A$ sur un corps valué (archimédien ou non) complet algébriquement clos, possédant une semi-norme spectrale $\|\cdot\|_{\text {sp }}$ et dont la norme est notée $\|\cdot\|$, on a trivialement.

$\|f\|_{s p}=\sup \left\{|\lambda|, \lambda \in \operatorname{sa}_{A}(f)\right\} \leqslant \sup \left\{|\lambda|, \lambda \in \operatorname{sp}_{A}(f)\right\} \leqslant \overline{\lim _{n \rightarrow \infty}} \sqrt[n]{\left\|f^{n}\right\|}$. Si $K=\mathbb{c}$, on sait que $A$ possède toujours la propriété "P" [2]. Nous allons voir maintenant grâce à l'exemple 2) que si $K$ est non archimédien, une K-algèbre de Banach $A$ ne possède pas toujours la propriété "P". 
Exemple 2. Soit $R \in] 0,1[$ et soit $D$ l'ensemble des $\xi \in K$ tels que $|\xi| \leqslant R$. Pour tout $h \in K(D)$, soit $E(h)$ l'ensemble des pôles de $h$ et soit $\Delta(h)$ l'ensemble des $\zeta \in U$ tels que $|\xi-a| \geqslant|a|$ quel que soit a $\epsilon E(h) \cap U$. Alors l'application

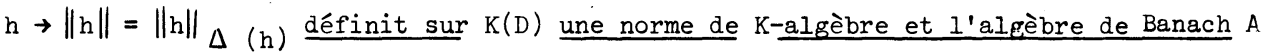
complétée de $K(D)$ pour cette norme admet une semi-norme spectrale $\|\cdot\|_{\text {sp }}$.

L'application identique $\times$ sur $D$ est un élément de classe algébrique tel que $\|x\|_{\text {sp }}=R<1=\lim _{n \rightarrow \infty}\left\|\mathrm{n} / \mathrm{xx}^{\mathrm{n}}\right\| ;$ de plus il existe un élément y $\in$ A tel que $\|y\|_{s p}=R<\sup \left\{|\lambda|, \lambda \in \operatorname{sp}_{A}(y)\right\}<\underset{n \rightarrow \infty}{\lim } \cdot \sqrt[n]{\left\|y^{n}\right\|}=1$.

L'exemple 3 montre que même si la propriété "P" est satisfaite, le spectre algébrique n'est pas nécessairement dense dans le spectre.

Exemple 3. Soit $D$ l'ensemble des $\xi \in K$ tels que $|\xi|<1$ et soit $\times$ l'application identique sur D. Soit $A=H(D) \cdot\{T\}$ une extension topologiquement pure de $H(D)$ de degré 1 . Alors l'algèbre $A$, dont la norme spectrale est la norme canonique possède la propriété " $P$ " et le spectre de X T dans A est égal à U tandis que son spectre algébrique est égal à D.

4. Caractérisation des algèbres de Krasner-Tate et de Tate parmi les algèbres de Banach.

Les résultats obtenus aux paragraphes précédents permettent de caractériser les algèbres de Krasner-Tate parmi les K-algèbres de Banach ultramétrique de la façon suivante :

Théorème 1 . Soit $\mathrm{A}$ une $\mathrm{K}$-algèbre de Banach dont la norme est notée $\|\cdot\|$, admettant une semi-norme spectrale notée $\quad\|\cdot\|_{\text {sp }}$. Alors A est une algèbre de Krasner-Tate si et seulement si A possède les propriétés suivantes :

a) A est noethérienne.

b) A est réduite.

c) $\|\mathrm{f}\|_{\mathrm{sp}} \in|\mathrm{K}|$ quel que soit $f \in A$;

a) pour tout élément $f \in A$ tel que $\|f\| \leqslant 1$, la suite $\left\|f^{n}\right\|$ est bornée ;

e) A contient une sous-K-algèbre $B$ dense dans $A$, principale, algébriquement de type fini.

Nous pouvons également caractériser les algèbres topologiquement de type fini réduites parmi les algèbres de Banach. On a les théorèmes 2 et 3 : 
Théorème 2. Une K-algèbre de Banach ultramétrique intègre admettant une semi-norme spectrale $\|\cdot\|_{\mathrm{sp}}$, dont la norme est notée $\|\cdot\|$, est topologiquement de type fini si et seulement si elle possède les propriétés c), d), e'), f) :

c) $\|f\|_{s p} \epsilon|K|$ quel que soit $f \in A$,

d) $\underline{\text { Si }}\|\mathrm{f}\|_{\mathrm{sp}} \leqslant 1$, la suite $\left\|\mathrm{f}^{\mathrm{r}_{1}}\right\|$ est bornée,

$\left.e^{\prime}\right)$ A contient une sous-K-algèbre de type fini sur $K$, dense dans $A$, dont le degré de transcendance sur $K$ est $\geqslant 1$,

f) A contient un élément t tel que A soit une extension entière de l'adhérence de $K[t] \underline{\text { dans }} A$.

Théorème 3. Une $\mathrm{K}$-algèbre de Banach ultramétrique réduite $\mathrm{A}$ admettant une semi-norme spectrale $\|\cdot\|_{\text {sp }}$ et dont la norme est notée $\|\cdot\|$, est topologiquement de type fini

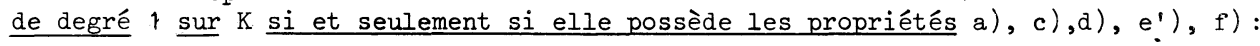

a) A est noethérienne,

c) $\|f\|_{s p} \in|K|$ pour tout $f \in A$.

d) $\underline{\text { Si }}\|f\|_{\text {sp }} \leqslant 1$, la suite $\left\|f^{n}\right\|$ est bornée.

$\left.e^{\prime}\right)$ A contient une sous-K-algèbre de type fini sur $K$, dense dans $A$ dont le degré de transcendance sur $K$ est $\geqslant 1$,

f) A contient un élément $t$ tel que $A$ soit une extension entière de l'adhérence de $K[t]$ dans $A$. 
1] $\mathrm{AMICE}, \mathrm{Y}$.

[2] BOURBAKI, N.

[3] BOURBAKI, N.

[4] ESCASSUT, A.

[5] ESCASSUT, A.

[6] ESCASSUT, A.

[7] Eseassut, A.

[8] GERRITZEN, L.

[9] GRAUERT, H. REMMERT, R.
Fonctions ultramétriques. Frontières analytiques dans certains quasi-connexes fermés d'un corps valué non archimédien complet et algébriquement clos. C.R.A.S. Paris, t. 268, p. 1251-1253 (28 mai 1969).

Espaces vectoriels topologiques. Chap. I, Hermann, Paris, 1953 (Actualité Scientifique et Industrielle).

Théorie spectrale. Chap. I, Hermann, Paris, 1967 (Actualité Scientifique et Industrielle).

Algèbres d'éléments analytiques au sens de Krasner. C.R.A.S., Paris, t. 270, p. 758-761 (23 mars 1970).

Algèbres de Banach d'éléments analytiques au sens de Krasner. Thèse de $3 e$ cycle, Fac. des Sciences de Bordeaux, 1970.

Complément sur le prolongement analytique dans un corps valué non archimédien complet algébriquement clos. C.R.A.S., Paris, t. 271, p. 718-721 (12 octobre 1970).

Algèbres de Krasner. C.R.A.S., Paris, t. 272, p. 598-601 (premier mars 1971).

Die Norm der gleichmäßigen Konvergenz auf reduzierten affinoiden Algebren. Journal für die reine und angewandte Mathemathematik Band 231n 1968.

Uber die Methode der diskret bewerteten Ringe in den nicht-archimedischen Analysis. inv. Math..t.2, p. 87-133, 1966. 
[10] GRAUERT, H.

[11] GRUSON, L.

[12] GRUSON, L.

[13] HOUZEL, C.

[14] KRASNER, M.

[15] LANG, S.

[16] LANG, S.

[17] LAZARD, M.

[18] MOTZKIN, E. ROBBA, $P$.

[19] REMMER, R.
Affinoide Uverdeckungen eindimensionaler, affinoīder

Räume. Presses Universitaires de France, Paris, I.H.E.S, Publications mathématiques, $n^{\circ} 34,1968$.

Algèbres de Banach ultramétriques (Journées PoitouAquitaine, Poitiers, 1967).

Fibrés vectoriels sur un polydisque ultramétrique.

Ann. Scient. Ec. Norm. Sup., 4 e série, t. 1, p. 45 à 89, 1968.

Espaces analytiques rigides sur un corps ultramétrique (d'après Tate). Colloque Poitou-Aquitaine, 1965.

Prolongement analytique uniforme et multiforme dans les corps valués complets. Colloque du C.N.R.S., ClermontFerrand, 1964.

Abelian varieties. Interscience tracts in pure and applied mathematics, number 7 . Interscience publischers, Inc. New-York, 1958.

Algebra, Addision Wesley. Publishing Company Inc. Reading Massachussetts, 1965.

Les zéros d'une fonction analytique. Presses Universitaires de France, Paris, I.H.E.S., Publications mathématiques $\mathrm{n}^{\circ} 14,1962$.

Ensembles satisfaisant au principe du prolongement analytique en analyse p-adique. C.R.A.S, Paris, t. 269, p. 126-129 (21 juillet 1969).

Algebraische Aspekte in der nichtarchimedischen analysis Proceeding of a conference on local fields. Nuffic Summer school held at Driebergen (The Netherlands) in 
Springer-Verlag Berlin, Heidelberg, New-York, 1967.

[20] ROBBA, P.

Fonctions analytiques sur les corps valués ultramétriques complets. Astérisque $n^{\circ} 10$, Octobre 1973.

[21] SALMON, P.

Série convergenti su un corpo non archimedeo con applicazione ai fasci analitici (Annali di Matematica pura ed applicata, serie IVT LXV, 1964).

[22] TATE, J. Rigid analytic spaces. Inv. Math. t. 12, fasc. 4, p. 257-289, 1971.

[23] ESCASSUT, A. Algèbres de Banach ultramétriques et algèbres de Krasner-Tate. Astérisque $n^{\circ} 10$, Octobre 1973.

\author{
Alain ESCASSUT \\ U.E.R. de Mathématiques \\ et d'informatique \\ Université de Bordeaux I \\ 351, cours de la Libération \\ 33405 - TALENCE
}

